\title{
MAKING \\ THE ELEMENTS IN THE UNIVERSE
}

n Karlheinz Langanke ${ }^{1}$ and Friedrich-Karl Thielemann ${ }^{2}$ - DOI: 10.1051/epn/2013304 [ ${ }^{1}$ GSI Helmholtz Center for Heavy Ion Research and Technical University Darmstadt, Germany

n ${ }^{2}$ Department of Physics, University of Basel, Switzerland

Nuclear Astrophysics combines astronomy/astrophysics with nuclear physics and aims at unveiling the origin of the chemical elements and the astrophysical sites where they are formed. Recent years have witnessed tremendous advances in powerful observatories, laboratory reaction measurements, radioactive ion-beam facilities providing highly unstable nuclei, and progress in astrophysical and nuclear modeling.

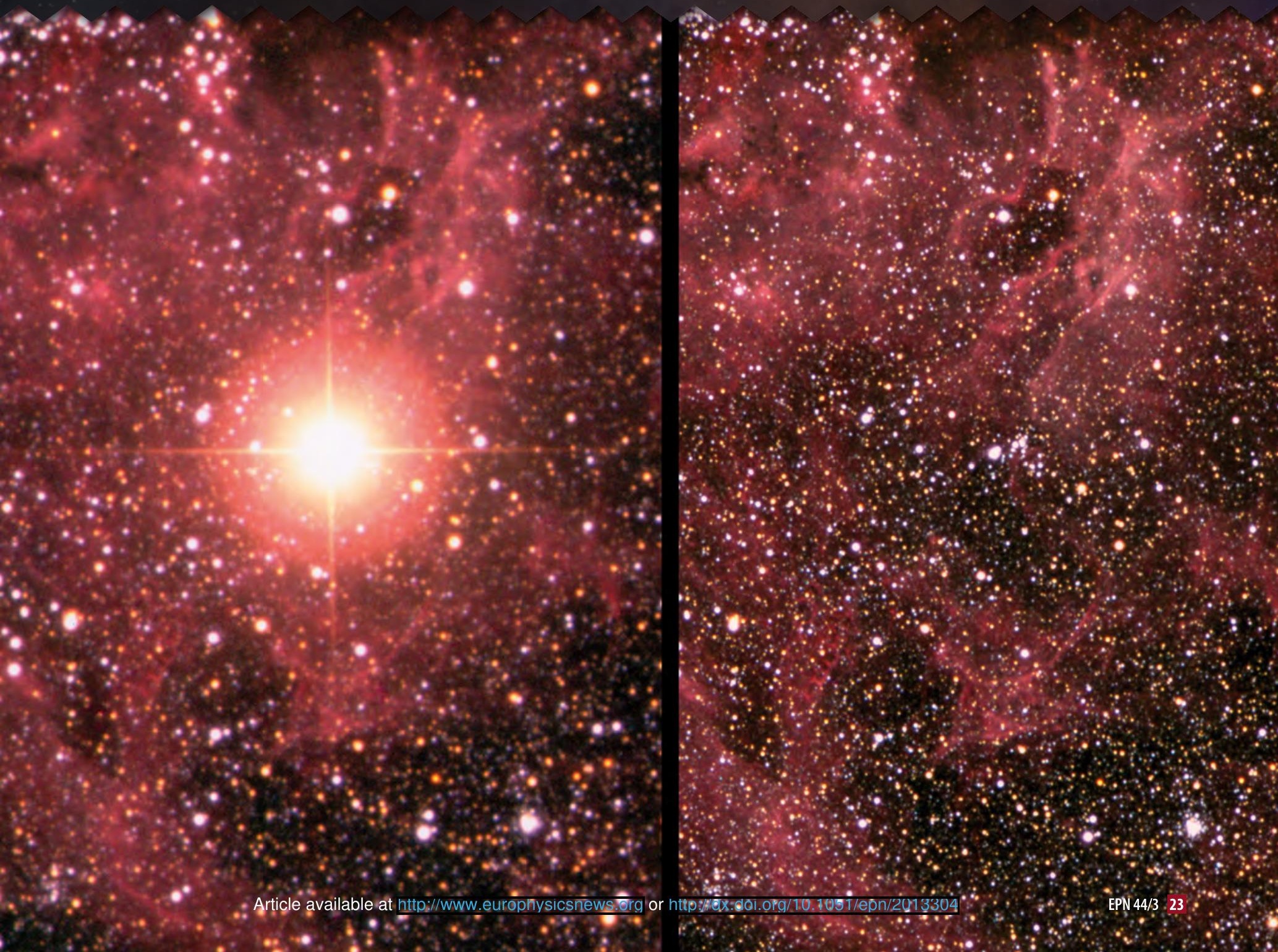


v FIG. 1: Solar abundances from $\mathrm{H}$ to $\mathrm{Bi}$ isotopes as a function of mass number $A$, spanning over 12 orders of magnitude [2]. For historical reasons Si is normalized to $10^{6}$. Only H, He, an Li were formed in the Big Bang, all heavier elements are due to stellar evolution and stellar explosions. Up to the Fe-peak

these are fusion reactions. The heavier elements are formed by neutron captures with peaks (red/blue shades) related to the closed neutron shells 50,82 , and 126 . The separation between

the peaks of the r(apid)-and the s(low) process is due to the fact that the reaction paths encounter these shell closures for different proton numbers Z (among stable or short-lived neutron-rich unstable nuclei, see also Fig.3).
N uclear Astrophysics has a long tradition, going back to the early days of nuclear physics, when nuclear reactions started to be considered as the source of energy generation in the stars. Later on, primordial nucleosynthesis during the Big Bang was understood to only have produced light nuclides (mainly ${ }^{1} \mathrm{H}$ and ${ }^{4} \mathrm{He}$ ). Because Big Bang densities at the relevant temperatures were too small to bridge the nuclear mass gaps at $A=5$ and 8 (for which no stable and only extremely short-lived nuclei exist), stars have been identified as the main cauldrons for the elements in the Universe. Already more than half a century ago, J. and M. Burbidge, W.A. Fowler, and F. Hoyle, as well as independently A. Cameron laid down the ground work to understand the origin of the abundance pattern of the elements, as we observe it today [1]. They postulated that stars go through various burning stages. The first stellar energy source is hydrogen fusion into helium, followed by further nuclear fusion processes, which involve the ashes of the previous stages up to nuclei around $\mathrm{Fe}$, possessing the highest binding energies. The origin of the heavier nuclei up to $\mathrm{Pb}$ and $\mathrm{Bi}$ or even $\mathrm{Th}, \mathrm{U}, \mathrm{Pu}$ and beyond is due to sequences of neutron captures and beta-decays.

\section{Stellar Evolution}

After enormous efforts in experimental and theoretical reaction investigations, required for nucleosynthesis studies (with initially assumed or approximated environment conditions), step by step a transition occurred to realistic astrophysical models. Such detailed stellar evolution models include all relevant nuclear physics as well as dynamics, radiation transport, mixing via convective instabilities or induced by rotation, and mass loss via stellar winds (for present models see e.g., [3]).

Stars with an original mass of less than $8 \mathrm{M}_{\Im}\left(\mathrm{M}_{9}\right.$ denotes the mass of the Sun) end their stellar evolution as white dwarfs (after having finished hydrogen and helium burning and losing significant amounts of mass in stellar winds which are observable as planetary nebulae). A white dwarf is the remaining central C/O-core of the star, which has typically the size of the Earth. It is very dense and stabilized by the pressure of the low-temperature degenerate Fermi gas of

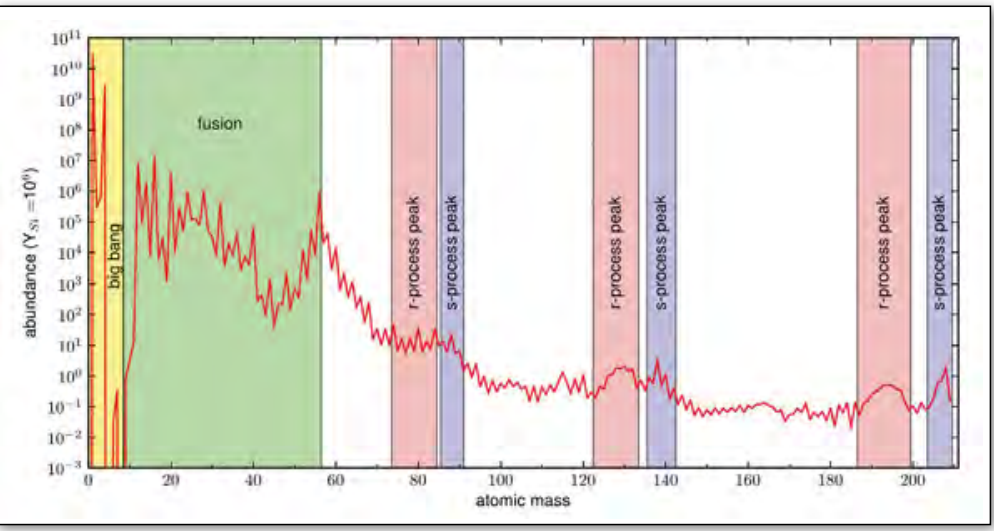

electrons, which prevents further contraction and burning stages. More massive stars pass through all nuclear burning stages, also encountering $\mathrm{C}, \mathrm{Ne}, \mathrm{O}$, and Si-burning and end with a central Fe-core. Consisting of matter with the highest binding energy per nucleon, no further nuclear burning can prevent this core to collapse up to nuclear densities. The collapse results in a central ultra-dense neutron star, and triggers the explosive ejection of the outer layers in an extremely bright event called a core collapse supernova (e.g., [4]).

\section{Supernovae}

Supernovae belong to the most powerful explosions in the Universe. They are also the dominant sources of intermediate and heavy elements $[6,7]$ and come spectroscopically in two varieties (type I and II, i.e., with or without hydrogen lines in their spectra), both similar in their output of kinetic energy close to $10^{44} \mathrm{~J}$.

Type Ia supernovae are related to the explosion of a white dwarf star, exceeding its maximum stable mass (the Chandrasekhar mass, $1.4 \mathrm{M}_{\Im}$ ) due to mass transfer from an orbiting companion star. This causes contraction and ignition of nuclear burning of $\mathrm{C}$ and $\mathrm{O}$ (mainly to intermediate mass nuclei up to the $\mathrm{Fe} / \mathrm{Ni}$ region) in an explosive manner. The nuclear burning front disrupts the whole star, leading to the ejection of about $0.6 \mathrm{M}_{9}$ of $\mathrm{Ni} / \mathrm{Fe}$, smaller amounts of intermediate mass elements from $\mathrm{Si}$ through $\mathrm{Ca}$, and some unburned $\mathrm{C}$ and $\mathrm{O}$ into the interstellar medium (e.g., [8]). As type Ia supernovae start from very similar initial conditions (a $1.4 \mathrm{M}_{3}$ white dwarf), they lead to similar light outbursts and can be utilized as standardizable light candles, thus also serving as distance indicators in the Universe. This property has led to the 2011 Nobel Prize discovery of the accelerating expansion of the Universe. Core-collapse supernovae (spectroscopically Type II or Ib/c, the latter having lost their hydrogen envelope) are related to the collapse of massive stars forming a neutron star, stabilized by the degeneracy pressure of nuclear matter. The gravitational binding energy of the stellar core, about $10^{46} \mathrm{~J}$, is released in form of neutrinos of all types, of which about $10^{44} \mathrm{~J}$ are converted into local thermal energy via neutrino and antineutrino captures on free neutrons and protons. This triggers, possibly together with the energy drawn from the winding of magnetic fields due to rotation and supplemented by hydrodynamic/convective instabilities, the ejection of the outer layers of the star with a total kinetic energy of about $10^{44} \mathrm{~J}$,similar to the one of type Ia supernovae [4]. The observation of 19 neutrinos from supernova SN1987A by the Kamiokande-II and the Irvine-Michigan-Brookhaven (IMB) water-Cerenkov detectors have proven that our basic understanding of core-collapse supernovae is correct. In spiral galaxies similar to the Milky Way they occur typically 2 to 3 times more often than type Ia supernovae.

This very brief and simplified description of the two types of supernova explosion mechanisms depends highly on the nuclear physics entering the understanding of such hot, 


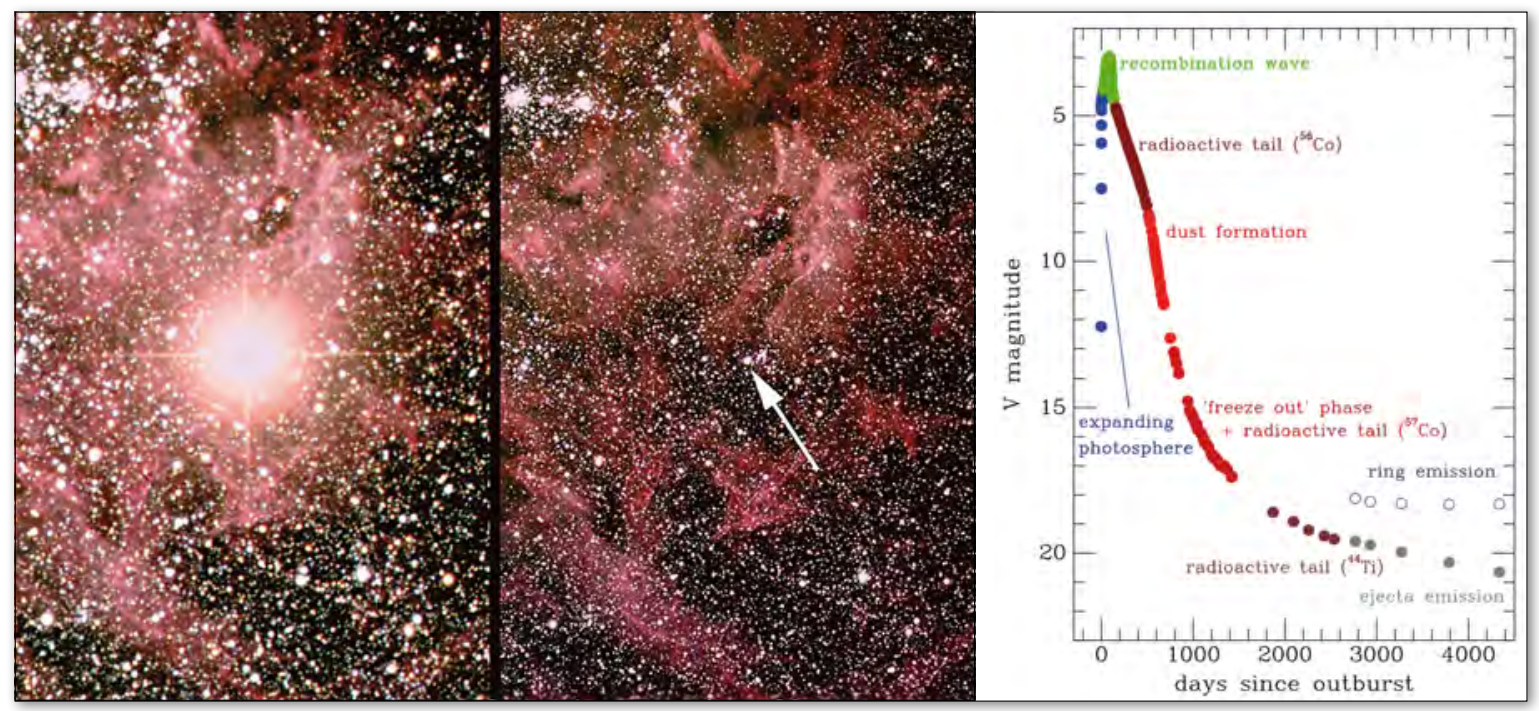

high-density plasmas. While nuclear burning through fusion is one important ingredient, reactions mediated via the weak interaction are essential for the dynamics of the collapsing core, the explosion and the associated explosive nucleosynthesis. Of particular importance during central burning of type Ia supernovae as well as in core-collapse supernovae are electron captures on protons (free and in nuclei), which are changed into neutrons. This process changes the overall proton/neutron ratio and reduces the electron degeneracy pressure and the core temperature, as the neutrinos produced by the capture can initially leave the star unhindered. The latter two effects accelerate the collapse of massive stars. There has been significant progress in recent years in describing electron captures under stellar conditions based on modern nuclear many-body models [9], which have been confirmed by recent measurements on selected nuclei [10]. The nuclear equation of state at and beyond nuclear densities enters the simulation of core collapse supernovae, the maximum neutron star mass, and the possible transition to black holes, combined with a transition from supernovae to hypernovae and Gamma-Ray Bursts [11].

\section{Properties of Nuclei far from Stability and Explosive Nucleosynthesis}

One of the great mysteries in nuclear astrophysics is the site of the so-called rapid neutron capture process ( $r$ process), which is responsible for the production of the heaviest elements up to Th, $\mathrm{U}$ and $\mathrm{Pu}[13]$. It requires an astrophysical environment with an extremely high neutron density, making neutron captures much faster than beta-decays. As a consequence the r-process runs through nuclei with such an extreme neutron excess that most of them have never been produced in the laboratory yet. Hence, r-process simulations rely on our theoretical understanding of nuclear structure far from stability. Of particular importance are nuclear masses and beta-decay half-lives, which determine the r-process path and duration, complemented by neutron capture cross sections, fission rates and yield distributions, and possibly neutrino-induced reaction cross sections [9]. The astrophysical site(s) of the r-process is/are not yet clearly identified. The current favorites are the neutrino-driven wind in core-collapse supernovae, and ejecta from neutron star mergers or supernovae arising from progenitor stars with fast rotation and strong magnetic fields.

Another topic is the synthesis of stable proton-rich isotopes, which cannot result from neutron capture processes. These can occur in a hot bath of photons during supernova explosions, where photodisintegrations combined with beta-decays can populate stable proton-rich isotopes ( $\mathrm{p}$-process). Another option is explosive hydrogen burning in binary stellar systems on the surface of white dwarfs (novae) or neutron stars (X-ray bursts, and also so-called superbursts), causing a rapid protoncapture process (rp-process). In both cases the properties of proton-rich unstable nuclei and their reaction cross sections are required (e.g., [15]), also addressed by experiments in radioactive ion beam (RIB) facilities and studies of proton and alpha-potentials in the related reactions.
4 FIG. 2:

(Left) Supernova 1987A; (Middle) the identified progenitor star (Sanduleak $\left.-69^{\circ} 202\right)$ in the Large Magellanic Cloud, a 20M star (Canglo Australian Observatory; (Right) the observed lightcurve (evolution of the brightness of the event with time), exhibiting the decayheat of unstable ${ }^{56} \mathrm{C}$, ${ }^{57} \mathrm{Co}$, and ${ }^{44} \mathrm{Ti}[5]$.
V FIG. 3: The nuclear chart $(\mathrm{N}, \mathrm{Z})$ with stable isotopes (black), unstable isotopes with known masses (green), or known half-lives (yellow). In order to produce the solar r-process component (rapid neutron capture) a very neutron-rich reaction path has to be encountered, which passes the relevant neutron shell closures at specific charge numbers Z (closest to stability with the longest beta-decay half-lives), in order to reproduce the peaks at $\mathrm{A}=130$ and 195 [12] (with courtesy from H. Schatz).

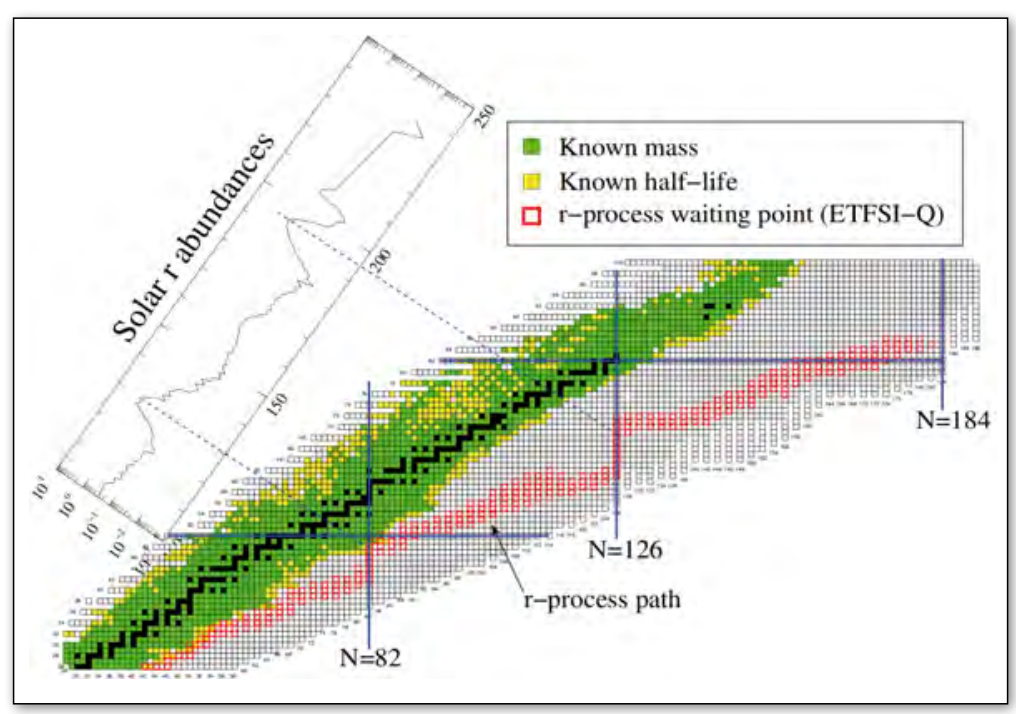




\section{Future Challenges}

Thanks to the collaborative effort of many researchers we have witnessed a significant progress in our quest to understand the origin of the elements in the Universe.Although we identified stars and their ultimate supernova explosions as the main element factories, several open questions still remain, defining the current major frontiers in nuclear astrophysics:

- the need of measurements of minute cross sections at the lowest possible energies, in particular by exploiting the unique possibility of cosmic-ray shielded underground laboratories like LUNA;

- the understanding of nuclear structure far from stability; - the understanding of reactions mediated by the weak interaction;

- the nuclear equation of state below and beyond nuclear densities, the maximum mass of neutron stars, and the formation of black holes in stellar collapse.

A new era in nuclear astrophysics will open once the next-generation radioactive ion beam (RIB) facilities in Asia, Europe and North America will be operational, removing much of the current nuclear uncertainties in astrophysical simulations, in particular for r-process nucleosynthesis. The flagship of the new facilities will be FAIR - the Facility of Antiproton and Ion Research which will serve as a next-generation RIB facility utilized within the NUSTAR collaboration. Moreover the CBM, PANDA and SPARC collaborations will shed new light on the phase diagram of nuclear matter and the nuclear Equation of State and determine important properties to plot of the magnetic field strength after the merger of two neutron stars in a binary stellar system

[14], which merge due to energy loss via gravitational wave radiation. The ejecta of the spiral arms are highly neutron-rich and cause a strong r-process during the expansion. (Right) color contour plot of the proton nucleon ratio indicating the neutron-richness of matter) from a fast rotating core collapse supernova with strong magnetic

fields (magnetar), which leads to similar r-process conditions in matter ejected along the rotation (z-)axis [13]. of FAIR as an international facility has started and first beams are expected for 2018 .

\section{About the authors}

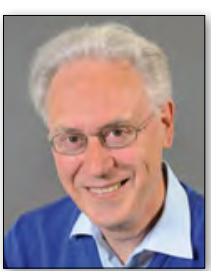

Karlheinz Langanke received his $\mathrm{PhD}$ at the University of Münster. He has been faculty member at the California Institute of Technology and the University of Aarhus. Currently he is professor at the TU Darmstadt and research director at the GSI Helmholtz Center for Heavy Ion Research, also Senior Fellow at the Frankfurt Institute for Advanced Studies.

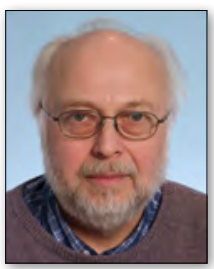

Friedrich-Karl Thielemann: $\mathrm{PhD}$ at TU Darmstadt / Max Planck Institute for Astrophysics, professorship at Harvard University 1986-1994, professor at the University of Basel since 1994, received the Hans A. Bethe Prize (APS) in 2008 and a Humboldt Research Award (spent at GSI) in 2009.

Langanke and Thielemann both served as Distinguished Visiting Scientists at Oak Ridge National Laboratory and were jointly awarded the Lise Meitner Prize 2012 of the EPS.

\section{References}

[1] E.M. Burbidge, G.R. Burbidge, W.A. Fowler, F. Hoyle, Rev. Mod. Phys. 29, 547 (1957); A.G.W. Cameron, Publ. Astron. Soc. Pac. 69, 201; Astron. J. 62, 9 (1957)

[2] M. Asplund, Ann. Rev. Astron. Astrophys. 43, 481 (2005)

[3] A. Heger, C.L. Fryer, S.E. Woosley, N. Langer, D.H. Hartmann, Astrophys. J. 591, 288 (2003)

[4] H.-T. Janka, Ann. Rev. Nucl. Part. Sci. 62, 407 (2012)

[5] B. Leibundgut, N.B. Suntzeff, Lecture Notes in Physics 598, 77 (2003)

[6] F.-K. Thielemann, K. Nomoto, M.-A. Hashimoto, Astrophys. J. 460, 408 (1996)

[7] F.-K. Thielemann, F. Brachwitz, P. Höflich, G. Martinez-Pinedo, K. Nomoto, New Astron. Rev. 48, 605 (2004)

[8] F.K. Röpke, M. Kromer, I.R. Seitenzahl et al.,Astrophys. J. 750, L19 (2012)
[9] K. Langanke, G. Martinez-Pinedo, Rev. Mod. Phys. 75, 81 (2003)

[10] A.L. Cole, T.S. Anderson, R.G.T. Zegers et al., Phys. Rev. C 86, 015809 (2012)

[11] J.M. Lattimer, Ann. Rev. Nucl. Part. Sci. 62, 485 (2012)

[12] H. Grawe, K. Langanke, G. Martinez-Pinedo, Rep. Progr. Phys. 70, 1525 (2007)

[13] C. Winteler, R. Käppeli, A. Perego et al., Astrophys. J 750, L22 (2012)

[14] D.J. Price, S. Rosswog, Science 312, 719 (2006)

[15] G. Gyürky, P. Mohr, Z. Fülöp et al., Phys. Rev. C 86, 041601 (2012)

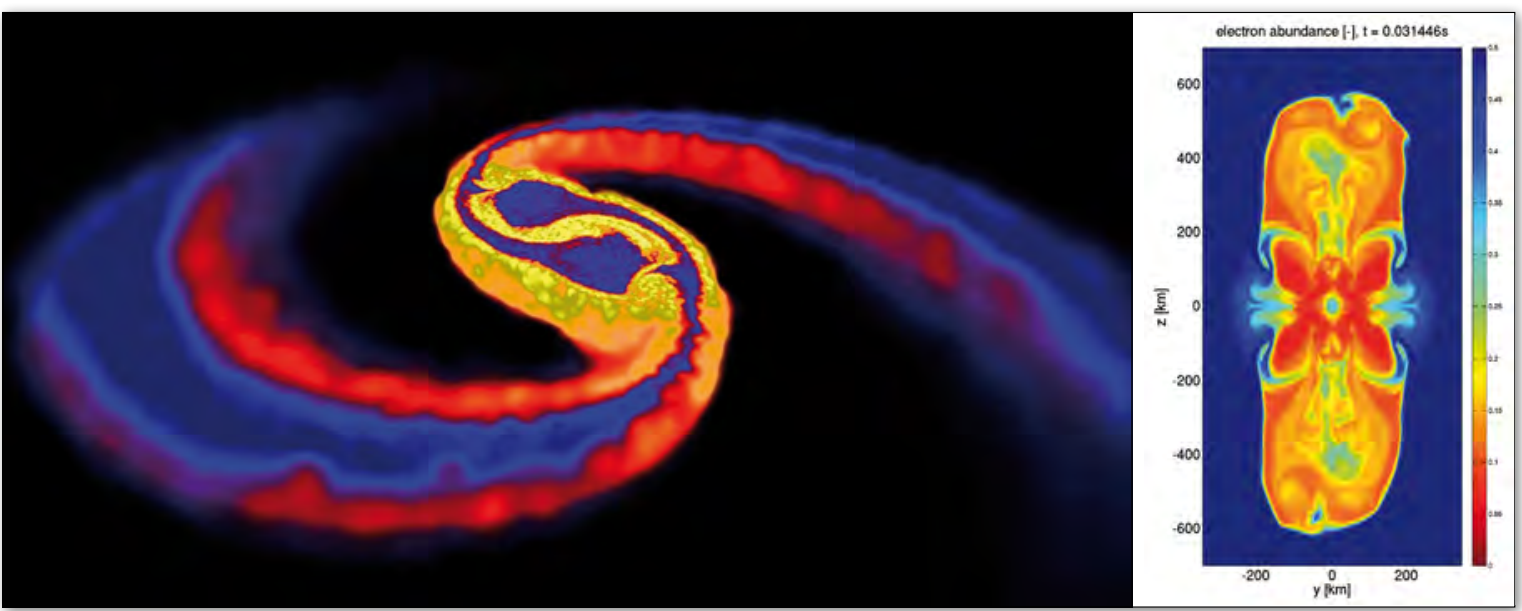

